\title{
Was können Sie Patienten mit Krampfadern empfehlen?
}

\author{
P. Stiefelhagen
}

\author{
Eine 42-jährige Patientin stellt sich in der Praxis vor und klagt \\ über Ödemneigung bei ausgeprägter Varikosis. Es findet sich eine \\ umschriebene Hyperpigmentierung i. S. einer leichten Stauungs- \\ dermatose. Die Patientin möchte wissen, ob und wie man die Varikosis \\ interventionell behandeln kann.
}

\begin{abstract}
Krampfadern sind häufig, doch die überwiegende Mehrzahl der Betroffenen hat nur leichte Beschwerden. Meist handelt es sich um eine primäre Varikosis als Folge einer Bindegewebsschwäche bzw. einer primären Venenklappeninsuffizienz. Eine sekundäre Varikosis kann nach einer tiefen Beinvenenthrombose oder bei einer Tumorkompression entstehen.
\end{abstract}

\section{Am Anfang steht der Ultraschall}

Die klinische Einteilung der chronischen Veneninsuffizienz erfolgt nach der CEAP(clinical condition, etiology, anatomic location, pathophysiology)-Klassifikation (Tab. 1). Auf Anamnese und klinische Untersuchung folgt die Duplexsonografie. Sie ist für die Therapieplanung unverzichtbar. Dabei geht es vorrangig um die Beurteilung der tiefen Beinvenen:

\section{Tab. 1 CEAP-Klassifikation der chronischen Veneninsuffizienz}

\begin{tabular}{|ll|}
\hline $\mathrm{C}_{0}$ & Keine sichtbaren Varizen \\
$\mathrm{C}_{1}$ & Besenreiser und retikuläre Varizen \\
$\mathrm{C}_{2}$ & Varizen $(>3 \mathrm{~mm})$ \\
$\mathrm{C}_{3}$ & Ödem \\
$\mathrm{C}_{4 \mathrm{a}}$ & Pigmentierung und Ekzem \\
$\mathrm{C}_{4 \mathrm{~b}}$ & Lipodermatosklerosis, Atrophie blanche \\
$\mathrm{C}_{5}$ & Abgeheiltes Ulkus \\
$\mathrm{C}_{6}$ & Offenes Ulkus \\
\hline
\end{tabular}

Sind sie offen und suffizient? Liegt ein Reflux in die oberflächlichen Venen vor?

\section{Konservative oder interventionelle Therapie?}

In den Stadien $\mathrm{C}_{\mathrm{O}}$ bis $\mathrm{C}_{3}$ steht die konservative Therapie im Vordergrund. Dazu gehören Kompressionsstrümpfe mit einem definierten Anlagedruck von ca. 20-30 mmHg. Im Unterschied zur medikamentösen Therapie ist bei den Kompressionsstrümpfen die Wirksamkeit belegt. Sie sind deshalb eine effektive Basistherapie. Bei den Medikamenten dürften sicherlich auch Placeboeffekte eine Rolle spielen.

Für die interventionelle Therapie stehen heute neben der Chirurgie (Crossektomie, Stripping, Phlebektomie) auch die chemische Ablation in Form einer Sklerotherapie und die endovenöse Katheterablation zur Verfügung. Letztere kann thermisch mit Laser oder Radiofrequenzapplikation, mechano-chemisch mit Clarivein oder mit einem Venenkleber (Venaseal) erfolgen. Die Wahl des Verfahrens ist abhängig von der Größe und Lage der Krampfadern. Der Goldstandard heute ist allerdings die endovenöse Therapie mit einer ultraschallgesteuerten Kathetereinlage. Bei der thermischen Ablation muss eine Tumeszenzanalgesie durchgeführt werden.

Vergleichende Untersuchungen haben gezeigt, dass die thermische Abla-

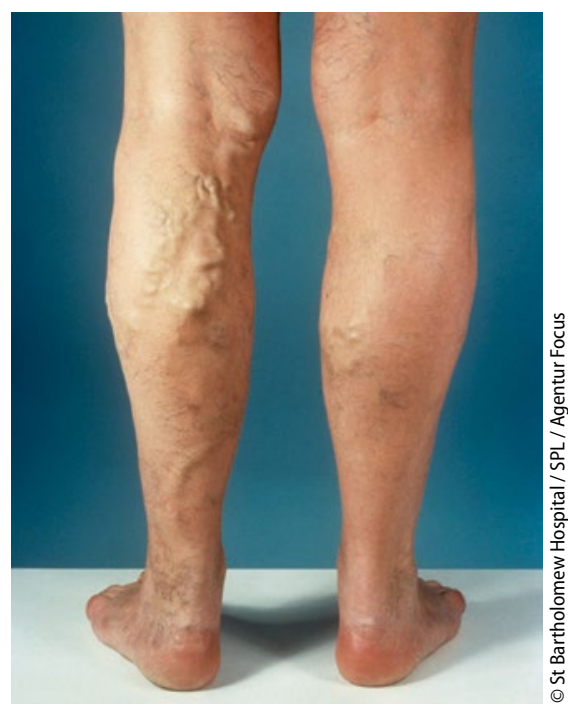

\section{Kasuistik}

\section{WIE GING ES WEITER?}

Bei der Patientin handelte es sich um ein Stadium $\mathrm{C}_{4 \mathrm{a}}$ nach der CEAP-Klassifikation. Sie wurde einem Phlebologen zugewiesen. Nach einer Duplexsonografie stellte er die Indikation für eine endovaskuläre Lasertherapie.

tion und die chirurgische Therapie bzgl. primärem und Langzeiterfolg durchaus vergleichbar sind, das endovenöse Verfahren aber sehr viel komplikationsärmer ist, d.h. Wundinfektionen treten um $70 \%$, Hämatome um $50-60 \%$ seltener auf. Auch können die Patienten drei bis fünf Tage früher ihre normale Aktivität wieder aufnehmen. Somit ist die Lasertherapie auch kosteneffektiver. Die Schaumsklerosierung ist weniger effektiv. Schwere Komplikationen wie Hämatome, Thrombosierungen und Entzündungen sind selten, aber durchaus möglich, und zwar bei allen invasiven Verfahren.

\footnotetext{
$\rightarrow$ Anschrift des Verfassers: Dr. med. Peter Stiefelhagen DRK-Klinikum Westerwald Krankenhaus Hachenburg D-57627 Hachenburg E-Mail:pdrstiefel@aol.com
} 